\title{
Correspondencia
}

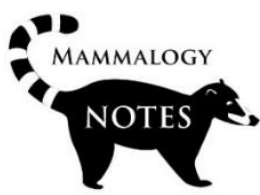

\section{Colección Teriológica de la Universidad de Antioquia (CTUA)}

\author{
Sergio Solarił* (iD), Alejandra Bonilla-Sánchez 1,2 (iD) \\ 1. Grupo Mastozoología Universidad de Antioquia, Facultad de Ciencias Exactas y Naturales, Universidad de Antioquia, Cl. 67 \\ \#53-108, Medellín, Colombia. \\ 2. Laboratório de Biologia Genômica e Molecular, Escola de Ciências da Saúde e a Vida, Pontifícia Universidad de Católica \\ do Rio Grande do Sul, Av. Ipiranga, 6681, Predio 12, Sala 134, Porto Alegre, Brasil. \\ * Correspondencia: sergio.solari@udea.edu.co
}

\section{Resumen}

La Colección Teriológica de la Universidad de Antioquia (CTUA) alberga más de 6.300 especímenes pertenecientes a 254 especies, 137 géneros, 40 familias y 13 órdenes, únicamente procedentes de Colombia, con una importante representatividad (48\% de la diversidad de especies mamíferas registrada para el país). Los órdenes mejor representados son Chiroptera, Rodentia y Didelphimorphia con 3.753, 1.391, y 267 especímenes, respectivamente. La colección cuenta con cuatro holotipos, correspondientes a Carollia colombiana, Saccopteryx antioquensis, Carollia monohernandezi, y Marmosops chucha. La CTUA ha sido empleada y referenciada en más de 60 trabajos de investigación.

Palabras clave: Antioquia, Diversidad, Investigación, Mastozoología, NW Colombia.

\begin{abstract}
The Colección Teriológica de la Universidad de Antioquia (CTUA) holds over 6.300 specimens belonging to 254 species, 137 genera, 40 families, and 13 orders, all of them from Colombia, with a relevant representation ( $48 \%$ of the mammalian species diversity recorded in the country). The orders better represented are Chiroptera, Rodentia, and Didelphimorphia with 3.753, 1.391, and 267 specimens, respectively. The collection holds three holotypes, corresponding to Carollia colombiana, Saccopteryx antioquensis, Carollia monohernandezi, and Marmosops chucha. The CTUA has been used and referenced in more than 60 research studies.
\end{abstract}

Key words: Antioquia, Diversity, Mammalogy, NW Colombia, Research

La colección Teriológica de la Universidad de Antioquia (CTUA) (Figura 1, 2) se encuentra asociada a la Facultad de Ciencias Exactas y Naturales en la sede principal de la Universidad de Antioquia ubicada en la ciudad de Medellín. Se considera que fue fundada en el año 2003, cuando se publicó un artículo en el Journal of Mammalogy (Woodman et al. 2003) que incluía un ejemplar de esta colección, aunque fue reportada bajo el código MUA (Museo de la Universidad de Antioquia). En la actualidad, la colección alberga aproximadamente 6.300 ejemplares de mamíferos, pertenecientes a 13 órdenes, 40 
familias, 137 géneros y 254 especies (Tabla 1). En total, 5.523 especímenes se encuentran catalogados y casi 800 están en proceso de ingreso a la colección. Los órdenes mejor representados son Chiroptera y Rodentia con 3.753 y 1.391 especímenes, respectivamente. Los especímenes proceden de los departamentos de Antioquia (4.357), Caldas (299), Bolivar (221), Chocó (219), La Guajira (130), Sucre (62), Casanare (50), Valle del Cauca (50), y Tolima (35), con una mayor representación del noroccidente de Colombia. Con excepción de 252 especímenes identificados a nivel de género, y uno a nivel de familia (Cricetidae), la mayoría de especímenes están identificados a especie. La colección resguarda los holotipos de tres especies de murciélagos y un marsupial: Carollia colombiana Cuartas, Muñoz \& González 2001, Saccopteryx antioquensis Muñoz \& Cuartas 2001, Carollia monohernandezi Muñoz, Cuartas-Calle \& González 2004, y Marmosops chucha Díaz-Nieto \& Voss 2016.

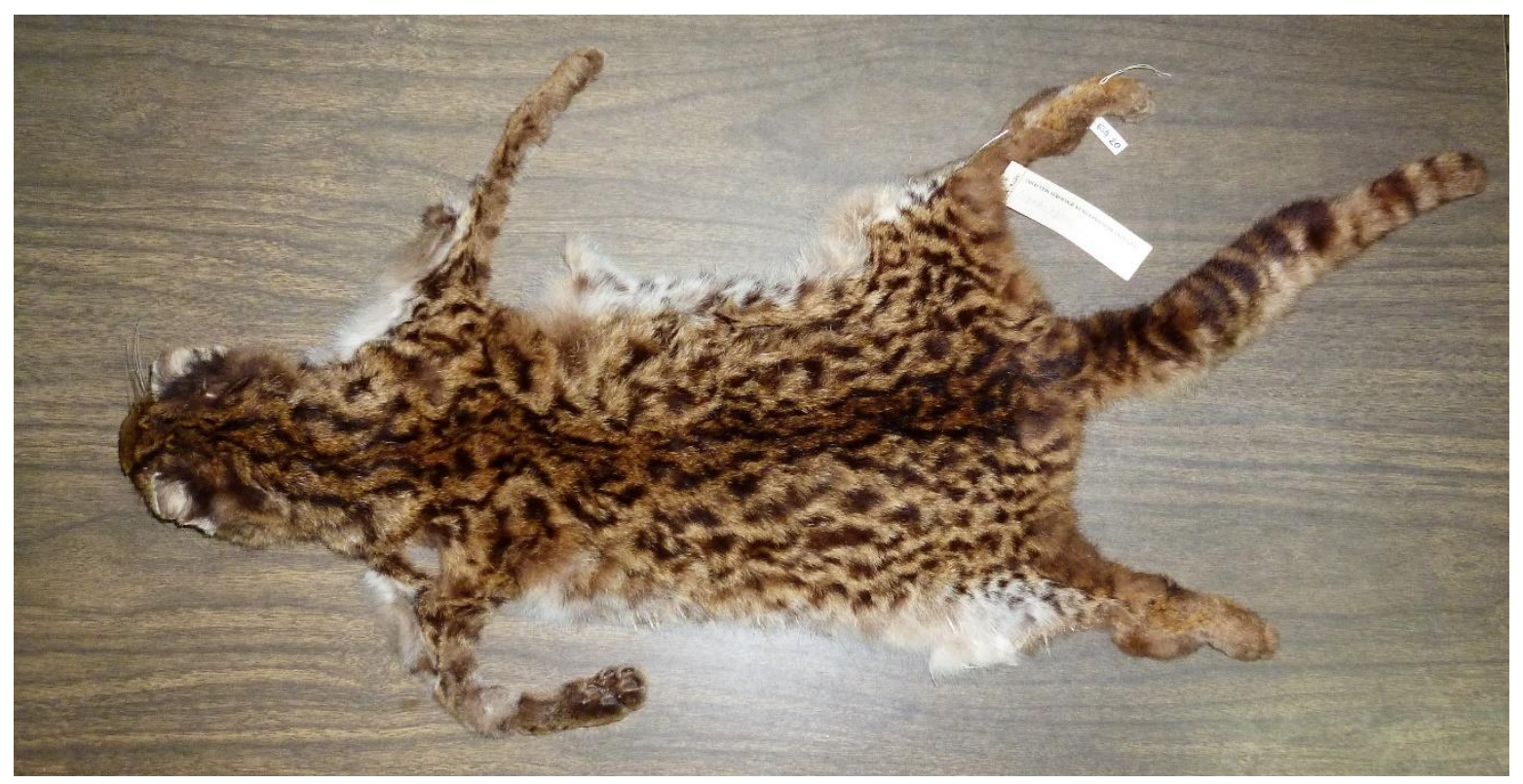

FIGURA 1. Detalles de los métodos de almacenamiento de material seco (piel de Leopardus tigrinus, tigrillo lanudo) en la Colección Teriológica de la Universidad de Antioquia (CTUA).

Los especimenes depositados en la CTUA han sido empleados en aproximadamente 60 publicaciones científicas. Estas abordan diferentes aspectos sobre la historia natural de mamíferos del país, como la dieta (Delgado \& Zurc 2007; Sánchez-Giraldo \& Díaz-N 2010) y la relación de esta con la forma del cráneo (Ospina-Garcés et al. 2016), autotomía caudal en roedores (Sánchez-Giraldo \& Delgado-V 2009), incluyendo revisiones y extensiones de distribución (Diaz-N \& Sánchez-Giraldo 2008; Martínez-Arias \& Solari 2013; Meza-Joya et al. 2018). Adicionalmente, ha sido referente para el estudio de la dieta de aves rapaces (Restrepo-Cardona et al. 2018). A nivel taxonómico, se han empleado especímenes en revisiones particulares para algunos grupos (Woodman et al. 2003; Voss et al. 2020), incluyendo la descripción de nuevas especies (Muñoz \& Cuartas 2001; Cuartas et al. 2001; Muñoz et al. 2004; Díaz-Nieto \& Voss 2016).

La información de esta colección ha contribuido para recopilaciones sobre estado de conocimiento de algunos grupos y especies en particular (Ramírez-Chaves et al. 2012; García-R et al. 2018), y ha sido la base para actualizar listados de especies a nivel de ecosistema como el bosque seco (Díaz-Pulido et al. 2014), departamental (Castaño 2012), y 
nacional (Solari et al. 2013). Así mismo, los especímenes han sido utilizados en el desarrollo de diferentes tesis de pregrado y posgrado que incluyen revisiones morfológicas y taxonómicas de pequeños mamíferos (Marín-C 2009; Estupiñán-Saavedra 2009; MartínezArias 2011; Ruiz \& Durán 2013; Cárdenas-González 2017; Morales-Martínez 2017; Cifuentes A 2018; Castañeda-L 2020).

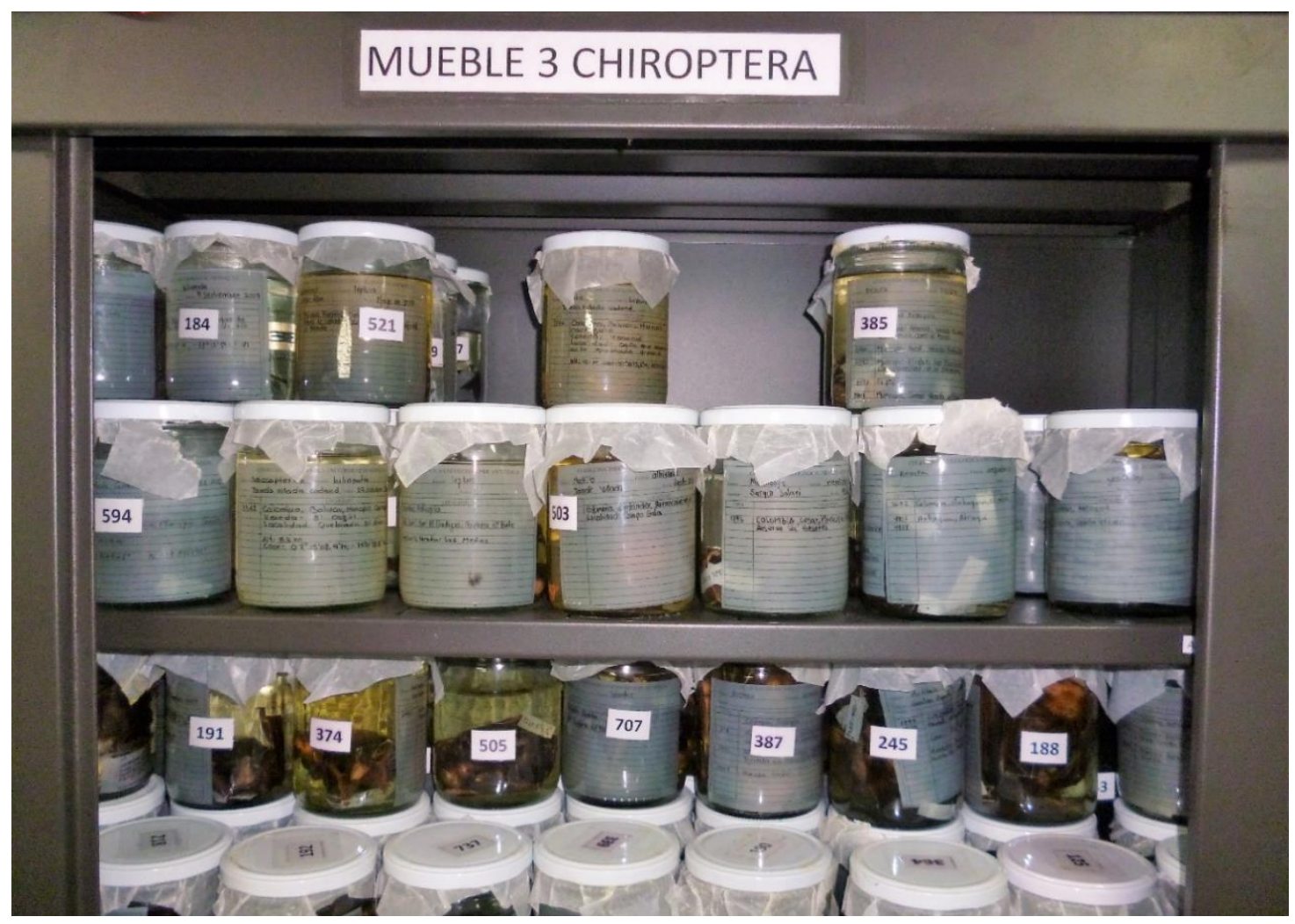

FIGURA 2. Detalles de los métodos de almacenamiento de material húmedo (animales completos en etanol 70\%) en la Colección Teriológica de la Universidad de Antioquia (CTUA).

La colección comenzó con los ejemplares colectados y depositados por Javier MuñozArango y Carlos Cuartas producto del desarrollo de diferentes cursos de Mastozoología, y los proyectos a nivel departamental y nacional sobre la diversidad de murciélagos (19792005). La mayoría de estos especímenes (ca. 1600) fueron registrados inicialmente como "MUA", pero actualmente están catalogados bajo el acrónimo CTUA. Posteriormente, a inicios de la década del 2000, estudiantes de pregrado del Instituto de Biología (Carlos Delgado-V., Alejandro Palacio, Danny Zurc, Juan Díaz-N., Camilo Sánchez-G., Sandra Ospina, entre otros), empezaron nuevas colectas en el Valle de Aburrá, retomando la idea de una colección biológica con énfasis en estudios sistemáticos de pequeños mamíferos. La ejecución de diferentes trabajos de grado y proyectos del Grupo Mastozoología de la Universidad de Antioquia, han contribuido al constante crecimiento de la colección. Igualmente, la efectiva comunicación con la Corporación Autónoma Regional de Antioquia, ha permitido el ingreso de ejemplares de mamíferos medianos y grandes, como primates y carnivoros, procedentes de atropellamientos o animales eutanasiados. Para los ejemplares depositados a partir del 2012, se ha tratado de garantizar la colecta y preservación de tejidos (fragmento de hígado en alcohol 70\%) de cada ejemplar, de 
manera tal que los ejemplares de la colección puedan ser empleados tanto para estudios morfológicos como genéticos y genómicos.

Desde sus inicios, el énfasis de esta colección ha sido el estudio sobre la taxonomía, sistemática y distribución de los pequeños mamíferos del noroccidente del país, la cual representa una de las zonas de mayor importancia biogeográfica, por su conexión con Centro América y el proceso del Gran Intercambio Biótico Americano (GABI, Webb 2006; Patterson et al. 2012). En la actualidad, esta colección es un centro de referencia para la fauna de mamíferos de esta región, incluyendo la actualización de áreas de distribución de especies endémicas, zonas de alta diversidad con prioridades de conservación, identificación de poblaciones nativas de especies focales (Botero et al. 2018; Zurc et al. 2018), así como el rol de los pequeños mamíferos en los ciclos de enfermedades zoonóticas (Londoño et al. 2017).

El desarrollo de la colección ha sido reforzado por el apoyo del Instituto de Biología y la Facultad de Ciencias Exactas y Naturales, que han permitido su reconocimiento como patrimonio natural de la Universidad de Antioquia, y su registro ante la Red Nacional de Colecciones (RNC) del Instituto Humboldt, con el número de registro 167. Esto ha favorecido mejoras en cuanto a los muebles para almacenamiento, ingreso de material desde otras instituciones, visitas por investigadores nacionales y extranjeros, la asignación de personal de apoyo (estudiantes de pregrado), y reconocimiento de la actividad curatorial por el docente a cargo del grupo y la colección.

TABLA 1. Órdenes, familias, géneros y especies de mamíferos depositados en la Colección Teriológica de la Universidad de Antioquia (CTUA).

\begin{tabular}{lccc}
\hline Orden & Familias & Géneros & Especies \\
\hline Chiroptera & 8 & 59 & 130 \\
Rodentia & 10 & 39 & 67 \\
Didelphimorphia & 1 & 9 & 18 \\
Carnivora & 5 & 13 & 16 \\
Pilosa & 4 & 4 & 5 \\
Primates & 4 & 4 & 5 \\
Artiodactyla & 2 & 2 & 3 \\
Paucituberculata & 1 & 1 & 2 \\
Cingulata & 1 & 2 & 2 \\
Lagomorpha & 1 & 1 & 2 \\
Eulipotyphla & 1 & 1 & 2 \\
Perissodactyla & 1 & 1 & 1 \\
Cetacea & 1 & 1 & 1 \\
Total & 40 & 137 & 254 \\
\hline
\end{tabular}

Al momento, la colección no cuenta con una página web con la información disponible al detalle, pero tanto esta como la base de datos (Excel) del catálogo se pueden consultar a través del curador de la colección. 


\section{AGRADECIMIENTOS}

El desarrollo y organización de la colección es el resultado de años de esfuerzo, a cargo de distintos investigadores y estudiantes que han contribuido incansablemente al crecimiento, mantenimiento, identificación, curación y organización del material biológico. Su compromiso está representado en cada nuevo aporte, y el reconocimiento académico de la colección es resultado de un correspondiente respaldo institucional, así como de la Facultad de Ciencias Exactas y Naturales, y la Vicerrectoría de Investigación de la Universidad de Antioquia.

\section{REFERENCIAS}

Botero-C S, Sánchez-L JD, Arias-Alzate A, Salazar E, Solari S. 2018. Felinos en los ecosistemas andinos de Antioquia. En: Quintero Vallejo E, Benavides AM, Moreno N, González-Caro S, editores. Bosques Andinos: estado actual y retos para su conservación en Antioquia. Medellín, Colombia: Fundación Jardín Botánico de Medellín Joaquín Antonio Uribe-Programa Bosques Andinos (COSUDE). p. 295-314.

Cárdenas-González C. 2017. Taxonomía y distribución del género de roedores andinos: Nephelomys (Cricetidae: Sigmodontinae) en Colombia. Tesis de Maestría. Universidad Nacional de Colombia, Bogotá, DC. Colombia.

Castañeda-L M. 2020. Evaluación de la variabilidad morfológica en Dermanura rava Miller 1902 (Chiroptera: Phyllostomidae) en Colombia. Tesis de Pregrado. Instituto de Biología, Universidad de Antioquia, Medellín. Colombia.

Castaño, JH. 2012. Mamíferos de Caldas: un análisis de vacíos de información. Boletín Científico Museo de Historia Natural Universidad de Caldas 16:101-119.

Cifuentes-A S. 2018. Acercamiento a la variación molecular de pequeños mamíferos no voladores en el norte de la Cordillera Central de Colombia. Tesis de Pregrado. Instituto de Biología, Universidad de Antioquia, Medellín. Colombia.

Cuartas, CA, Muñoz J, González M. 2001. Una nueva especie de Carollia Gray, 1838 (Chiroptera: Phyllostomidae) de Colombia. Actualidades Biológicas 23(75):63-73.

Delgado-V C, Zurc D. 2005. New records of Olallamys albicauda (Rodentia: Echimyidae) in Antioquia, Colombia. Brenesia 63-64:131-132.

Delgado-V C, Zurc D. 2007. Diet of the Crab-eating fox Cerdocyon thous (Carnivora: Canidae) in the Paramo de Belmira, Antioquia, Colombia. Brenesia 67:73-74.

Díaz-N JF, Sánchez-Giraldo C. 2008. Notable altitudinal range extension of the Northern nakedtailed armadillo Cabassous centralis (Cingulata: Dasypodidae) in Colombia. Brenesia 69:7576.

Díaz-N, JF, Gómez-Laverde M, Sánchez-Giraldo C. 2011. Rediscovery and redescription of Marmosops handleyi (Pine 1981) (Didelphimorphia: Didelphidae), the least known Andean opossum. Mastozoología Neotropical 18:45-61.

Díaz-Nieto JF, Voss RS. 2016. A revision of the didelphid marsupial genus Marmosops, Part 1. Species of the subgenus Sciophanes. Bulletin of the American Museum of Natural History 402:1-70

Díaz-Pulido A, Benítez A, Gómez-Ruiz DA, Calderón-Acevedo CA, Link A, Pardo A, Forero F, de Luna AG, Payan E, Solari S. 2014. Mamíferos del Bosque Seco, una mirada al Caribe Colombiano. En: Pizano C, García H, editores. El Bosque Seco Tropical en Colombia. Bogotá, DC, Colombia: Instituto de Investigación de Recursos Biológicos Alexander von Humboldt (IAvH). p. 129165.

Mammalogy Notes 2020, 6(2), 191; https://doi.org/10.47603/mano.v6n2.191 
Estupiñán-Saavedra E. 2009. Revisión preliminar de la morfología y sofometría de las musarañas del grupo Cryptotis thomasi (Soricomorpha: Soricidae) de la cordillera oriental de Colombia. Tesis de Pregrado. Escuela de Biologia, Universidad Industrial de Santander, Bucaramanga. Colombia.

García- R S, Montilla SO, Bustamante-Manrique V, Bustamante-Manrique S, Cepeda-Duque C, Sánchez Londoño JD, Ramírez-Chaves HE. 2018. Estado de la investigación primatológica en el eje cafetero y Antioquia, Colombia. Neotropical Primates 24(2):56-63.

Londoño AF, Acevedo-Gutiérrez LY, Marín D, Contreras V, Díaz FJ, Valbuena G, Labruna MB, Hidalgo M, Arboleda M, Mattar S, Solari S, Rodas JD. 2017. Wild and domestic animals likely involved in rickettsial endemic zones of Northwestern Colombia. Ticks and Tick-borne Diseases 8:887894.

Marín-C D. 2009. Caracterización morfológica y morfométrica de las musarañas de orejas pequeñas del género Cryptotis (Mammalia: Soricidae) en la Cordillera Central Colombiana. Tesis de Pregrado. Instituto de Biología, Universidad de Antioquia, Medellín. Colombia.

Marín-C D, Ramírez-Chaves HE, Suárez-C AF. 2012. Revisión cráneo-dentaria de Procyon (Carnivora: Procyonidae) en Colombia y Ecuador, con notas sobre su taxonomía y distribución. Mastozoología Neotropical 19:259-270.

Marin-C D, Corrales JD, Valencia JD. 2018. Nuevos registros de la rata cangrejera Ichthyomys hydrobates (Winge, 1891) (Rodentia: Cricetidae) en Colombia. Mastozoología Neotropical 25:235-243.

Martínez-Arias, V. 2011. Taxonomía de Sturnira lilium y Sturnira ludovici (Chiroptera: Phyllostomidae) para Colombia. Tesis de Pregrado. Instituto de Biología, Universidad de Antioquia, Medellín. Colombia.

Martínez-Arias, VM, Solari S, Diaz-N JF. 2010. Re-evaluation of a Colombian record of Sturnira thomasi de la Torre and Schwartz 1966 (Phyllostomidae: Stenodermatinae). Chiroptera Neotropical 16:604-610.

Martínez-Arias VM, Solari S. 2013. Extensión del área de distribución de Sturnira koopmanhilli McCarthy et al. 2006 en Colombia. Therya, 4(3):617-625. https:// doi.org/10.12933/therya-13152

Meza-Joya FL, Ramos E, Cediel F, Martínez-Arias V, Colmenares J, Cardona D. 2018. Predicted distributions of two poorly known small carnivores in Colombia: the greater grison and striped hog-nosed skunk. Mastozoología Neotropical 25(1):89-105. https:/ / doi.org/10.31687/ saremMN.18.25.1.0.09

Morales-Martínez DM. 2017. Taxonomía y sistemática de los murciélagos del género Micronycteris Gray, 1982 (Chiroptera: Phyllostomidae) en Colombia. Tesis de Maestría. Departamento de Biología, Universidad Nacional de Colombia, Bogotá, DC. Colombia.

Muñoz J, Cuartas CA. 2001. Saccopteryx antioquensis n. sp. (Chiroptera: Emballonuridae) del noroeste de Colombia. Actualidades Biológicas 23(75):53-61.

Muñoz J, Cuartas-Calle CA, González M. 2004. Se describe una nueva especie de murciélago del género Carollia Gray, 1838 (Chiroptera: Phyllostomidae) de Colombia. Actualidades Biológicas 26(80):80-90.

Ospina-Garcés SM, De Luna E, Herrera-M LG, Flores-Martínez JJ. 2016. Cranial shape and diet variation in Myotis species (Chiroptera: Vespertilionidae): testing the relationship between form and function. Acta Chiropterologica 18(1):163-180. https:// doi.org/10.3161/15081109ACC2016.18.1.007 
Patterson BD, Solari S, Velazco PM. 2012. The role of the Andes in the diversification and biogeography of Neotropical mammals. En: Patterson BD, Costa LP, editores. Bones, clones, and biomes: the history and geography of recent Neotropical mammals. Chicago, IL., EE.UU. The University of Chicago Press. p. 351-378.

Ramírez-Chaves HE, Mulder KP, Marín D, Pérez WA, Martínez-Arias VM. 2012. Has Colombian Weasel Mustela felipei been overlooked in collections? Small Carnivore Conservation 47:30-34.

Ramírez-Chaves HE, Noguera-Urbano EA, Morales-Martínez DM, Zurc D, Vargas-Arboleda AF, MantillaMeluk H. 2020. Endemic bats (Mammalia: Chiroptera) of Colombia: State of knowledge, distribution, and conservation. Universitas Scientiarum 25(1):55-94. https://doi.org/10.11144/Javeriana.SC25-1.ebmc

Restrepo-Cardona JS, Marín-C D, Sánchez-Bellaizá DM, Rodríguez-Villamil DR, Berrío S, Vargas L, Mikkola H. 2018. Diet of Barn Owl (Tyto alba), Spectacled Owl (Pulsatrix perspicillata) and Rufous-banded Owl (Strix albitarsis) in the western Andes of Colombia. Ornitología Neotropical 29:193-198.

Reyes-Amaya N, Lozáno-Flórez J, Flores D, Solari S. 2016. Distribution of the Spix's disk-winged bat, Thyroptera tricolor Spix, 1823 (Chiroptera: Thyropteridae) in Colombia, with first records for the middle Magdalena Valley. Mastozoología Neotropical. 23(1):127-137.

Rodríguez-Posada ME, Ramírez-Chaves HE. 2012. Distribución, morfología y reproducción del murciélago rayado de orejas amarillas Vampyriscus nymphaea (Mammalia: Chiroptera) en Colombia. Acta Zoológica Mexicana. 28(2):341-352.

Ruíz G, Durán C. 2013. Análisis morfológico y morfométrico del complejo de especies Anoura caudifer (Chiroptera: Glossophaginae) en Colombia. Tesis de Pregrado. Facultad de Ciencias Naturales, Exactas y de la Educación, Universidad del Cauca, Popayán. Colombia.

Sánchez-Giraldo C, Delgado-V CA. 2009. Dos nuevos casos de autotomía caudal en roedores suramericanos. Mastozoología Neotropical 16:481-484.

Sánchez-Giraldo C, Díaz-N JF. 2010. Hábitos alimenticios de Heteromys australis (Rodentia: Heteromyidae) en el norte de la Cordillera Central de Colombia. Mastozoología Neotropical 17:189-194.

Sánchez-Londoño JD, Gómez-R DA, Solari S, Molina A. 2019. Mamíferos (Mammalia) de la Reserva Forestal Protectora Alto de San Miguel (Caldas-Antioquia, Colombia). En: Sánchez-Londoño JD, Tuberquia DJ, Parra JL, editores. Estudios en Biodiversidad del Alto de San Miguel. Medellín, Colombia: Editorial Universidad CES. p. 69-89.

Solari S, Muñoz-Saba Y, Rodríguez-Mahecha JV, Defler T, Ramírez-Chaves H, Trujillo F. 2013. Diversidad, Endemismo y Conservación de los Mamíferos de Colombia. Mastozoología Neotropical 20:301-365.

Voss RS, Giarla TC, Díaz-Nieto JF, Jansa SA. 2020. A revision of the didelphid marsupial genus Marmosa. Part 2, Species of the rapposa group (subgenus Micoureus). Bulletin of the American Museum of Natural History 439:1-60. https:/ / doi.org/10.1206/0003-0090.439.1.1

Webb SD. 2006. The Great American Biotic Interchange: patterns and processes. Annals of the Missouri Botanical Garden 93:245-257.

Woodman N, Cuartas-Calle CA, Delgado-V CA. 2003. The humerus of Cryptotis colombiana and its bearing on the species' phylogenetic relationships (Soricomorpha: Soricidae). Journal of Mammalogy 84(3):832-839. https:/ / doi.org/10.1644/BME-007

Zurc D, Velazco PM. 2010. Análisis morfológico y morfométrico de Carollia colombiana Cuartas et al. 2001 y C. monohernandezi Muñoz et al. 2004 (Phyllostomidae: Carollinae) en Colombia. Chiroptera Neotropical 16(1):549-67. 
Zurc D, López-Castañeda C, Solari S. 2018. Murciélagos de bosques andinos de Antioquia. En: Quintero Vallejo E, Benavides AM, Moreno N, González-Caro S, editores. Bosques Andinos: estado actual y retos para su conservación en Antioquia. Medellín, Colombia: Fundación Jardín Botánico de Medellín Joaquín Antonio Uribe-Programa Bosques Andinos (COSUDE). p. 269-292.

Editor: Miguel E. Rodríguez-Posada Recibido 2020-09-10

Revisado 2020-09-15

Aceptado 2020-10-12

Publicado 2020-12-06 